\title{
Insetos predadores em copas de Citrus deliciosa (Rutaceae) sob manejo orgânico no sul do Brasil
}

\author{
Rosana M. de Morais ${ }^{1}$, Aline Barcellos ${ }^{2} \&$ Luiza R. Redaelli ${ }^{1,3}$
}

1. Programa de Pós-graduação em Biologia Animal, Universidade Federal do Rio Grande do Sul, Av. Bento Gonçalves, 9500, Bloco IV, Prédio 43435, 91501-970 Porto Alegre, RS, Brasil. (entomorais@yahoo.com.br)

2. Museu de Ciências Naturais, Fundação Zoobotânica do Rio Grande do Sul, Rua Dr. Salvador França, 1427, 90690-000 Porto Alegre, RS, Brasil. (alinebar@fzb.rs.gov.br)

3. Departamento de Fitossanidade, Faculdade de Agronomia, UFRGS, Av. Bento Gonçalves, 7712, 91540-000 Porto Alegre, RS, Brasil. (luredael@ufrgs.br)

\begin{abstract}
Predatory insects in canopies of Citrus deliciosa (Rutaceae) under organic management in southern Brazil. The composition, abundance and diversity of predatory insects occurring on canopies of Citrus sinensis Tenore var. Montenegrina, in Montenegro, State of Rio Grande do Sul, Brazil, were studied from March 2004 to March 2005. A total of 658 predaceous insects were collected, representing 51 species from ten families and five orders, Coleoptera, Hymenoptera, Neuroptera, Thysanoptera and Hemiptera. The most abundant species were Camponotus sp. 1 (Hymenoptera, Formicidae) (16.87\%) and Coccidophilus sp. (Coleoptera, Coccinellidae) $(11.85 \%)$. The highest abundance, richness and evenness of predatory insects were recorded in spring, although no significant difference among seasons has been found.
\end{abstract}

KEYWORDS. Citrus, diversity, Insecta, predation, species richness.

RESUMO. A fauna de insetos predadores em copas de Citrus deliciosa Tenore var. Montenegrina, em Montenegro, Rio Grande do Sul, Brasil, foi avaliada quanto à sua composição, abundância e diversidade, entre março de 2004 e março de 2005. Foram coletados, no total, 658 insetos predadores, representados por 51 espécies de dez famílias e cinco ordens, Coleoptera, Hymenoptera, Neuroptera, Thysanoptera e Hemiptera. As espécies mais abundantes foram Camponotus sp. 1 (Hymenoptera, Formicidae) (16,87\%) e Coccidophilus sp. (Coleoptera, Coccinellidae) (11,85\%). A maior abundância, riqueza e eqüitabilidade de insetos predadores foi registrada na primavera, embora não tenha sido constatada diferença significativa entre as estações.

PALAVRAS-CHAVE. Citros, diversidade, Insecta, predação, riqueza de espécies.

Insetos predadores têm sido apontados como importantes fatores de regulação populacional (RICKLEFS \& Miller, 1999). Em uma avaliação dos agentes entomófagos, os predadores, comparativamente aos parasitóides, apresentam atributos mais favoráveis, como destruir um grande número de presas durante seu desenvolvimento (Berti Filho \& Ciociola, 2002). Tais organismos constituem a principal força de regulação na dinâmica de populações de muitas espécies de presas (Pedigo, 1996), podendo ser encontrados em quase todos os hábitats, tanto naturais como agrícolas, fazendo parte de inúmeras cadeias alimentares. Em agroecossistemas, estes grupos são investigados por ser o estudo das interações predador-presa base da teoria e prática do controle biológico (BERRYMAN \& GUTIERREZ, 1999).

O citros, por ter caráter perene, pode ser considerado como uma cultura que abriga grande diversidade de insetos benéficos (LARA et al., 1977). Este fato pode ser evidenciado pelos levantamentos de espécies de coccinelídeos nesta cultura, desenvolvidos no Brasil por Arioli \& Link (1987), Silva et al. (2001) e WolfF et al. (2004). Em relação a comunidades de insetos predadores associados ao citros, entretanto, poucos trabalhos foram desenvolvidos, destacando-se o de Elizondo Solis (2002), realizado na Costa Rica. Neste trabalho, objetiva-se ampliar o conhecimento da fauna de insetos predadores associados ao citros, em termos de sua composição, abundância e diversidade, bem como de aspectos relacionados à sua sazonalidade, em um pomar mantido sob cultivo orgânico no sul do Brasil.

\section{MATERIAL E MÉTODOS}

O trabalho foi desenvolvido em um pomar de tangerineiras da variedade Montenegrina (Citrus

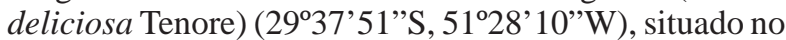
município de Montenegro, Rio Grande do Sul. O clima desta região, segundo a classificação de Köppen, é do tipo Cfa, subtropical úmido com precipitações bem distribuídas no ano, sofrendo a influência de massas de ar tropicais e polar-atlânticas. A temperatura média anual é de $19,1^{\circ} \mathrm{C}$, com a máxima média de $25,8^{\circ} \mathrm{C}$, mínima média de $14,4^{\circ} \mathrm{C}$ e a precipitação média é de $1.424 \mathrm{~mm} / \mathrm{ano}$ (IPAGRO, 1989). A área pertence à Depressão Central do Estado, apresentando topografia levemente ondulada, com menos de $100 \mathrm{~m}$ de altitude.

O pomar possui uma área de 0,6 ha e aproximadamente 300 plantas com 14 anos de idade. Desde sua instalação, vem sendo mantido sob manejo orgânico, com aplicações de chorume e composto provenientes da usina de compostagem da Cooperativa dos Citricultores Ecológicos do Vale do Caí (ECOCITRUS). Como tratamentos fitossanitários, são utilizadas calda bordalesa, três vezes por ano, e calda sulfocálcica, anualmente. Plantas espontâneas presentes nas entrelinhas são controladas apenas com roçadas anuais. 
As amostragens foram realizadas quinzenalmente, no período de 29 de março de 2004 a 14 de março de 2005, totalizando seis ocasiões amostrais no outono de 2004, sete no inverno de 2004, seis na primavera de 2004 e sete no verão de 2005. Em cada ocasião, 24 plantas foram previamente sorteadas e, destas, retirada uma unidade amostral de ambas as faces da copa voltadas para as entrelinhas, totalizando 48 unidades por ocasião. Para a retirada das unidades amostrais, utilizou-se um pano-debatida, de cor branca, medindo $1 \mathrm{~m}^{2}$, o qual era disposto sob a copa da planta e, com auxílio de um bastão de madeira, efetuavam-se dez batidas sobre um ramo central da copa. Imediatamente, o pano era enrolado e sacudido dentro de um saco plástico devidamente etiquetado. Em laboratório, os insetos foram separados e acondicionados em álcool $70 \%$ para posterior identificação. Esta foi realizada, inicialmente, em nível de ordem e família, com auxílio de bibliografia e, posteriormente, aqueles pertencentes a famílias que incluem espécies com hábito predador foram enviados a especialistas para identificação em nível genérico e específico. Os dados meteorológicos de temperaturas máxima, média, mínima e precipitação foram obtidos junto à Estação Experimental da Fundação Estadual de Pesquisa Agropecuária (FEPAGRO), situada no município de Taquari, RS.

A partir das espécies obtidas em cada uma das ocasiões de amostragem, foi construída uma curva de suficiência amostral utilizando-se o programa estatístico EstimateS versão 7.5 (Colwell, 2005). Por meio deste programa, também foram obtidos os valores estimados para o esforço amostral, sendo utilizados os estimadores: Bootstrap, que se baseia na proporção de unidades amostrais em que cada espécie esteve presente, e Chao 2, no número de espécies registradas em uma ou duas ocasiões durante todo o período amostral (MoRENo, 2001; MAGURRAn, 2004).

As estações do ano foram comparadas quanto à abundância e riqueza de espécies através de curvas de rarefação, utilizando-se os programas BioDiversity professional versão 2 (MCALEECE, 1997) e Past versão 1.34 (HAMmER et al., 2001). A correlação entre a abundância de indivíduos e os fatores abióticos de temperatura e precipitação pluviométrica foi analisada através do coeficiente de correlação de Pearson, pelo programa Past. A constância (c) foi calculada segundo SILVEIRA-NETo et al. (1976), sendo as espécies categorizadas em constantes (c> $50 \%)$, acessórias $(25 \leqslant c \leqslant 50 \%)$ e acidentais (c $<25 \%)$. As espécies dominantes foram classificadas em uma escala com base na abundância relativa (ar), assim convencionadas: rara, ar $\leqslant 0,01$; intermediária, $0,01<\operatorname{ar} \leqslant 0,1$; dominante, ar $>0,1$.

Os espécimens amostrados estão depositados no Departamento de Fitossanidade da Faculdade de Agronomia da Universidade Federal do Rio Grande do Sul (UFRGS).

\section{RESULTADOS E DISCUSSÃO}

Ao longo de 26 ocasiões de amostragem, foram coletados 658 insetos com hábito predador, distribuídos em cinco ordens e dez famílias, totalizando 51 espécies (Tab. I). Tais dados resultaram em uma curva de suficiência amostral que demonstra uma redução na inclinação a partir do final da primavera, devido à desaceleração no registro de novas espécies (Fig. 1), sugerindo que o número obtido de espécies provavelmente esteja próximo da riqueza existente na área de estudo. De acordo com o estimador Bootstrap, $88,1 \%$ das espécies esperadas foram amostradas, enquanto Chao 2 estimou em apenas $70,1 \%$.

Com relação às ordens, Hymenoptera foi a que registrou maior número de indivíduos $(51,2 \%)$, todos pertencentes à família Formicidae. As formigas são consideradas como os mais abundantes e importantes predadores, principalmente nas plantas jovens e vegetação de pequeno porte (HOLLDOBLER \& WILSON, 1990). Os formicídeos exercem alta pressão de predação sobre artrópodes de menor mobilidade e tamanho, especialmente em copas, onde são dominantes em relação a espécies de formigas não-predadoras (Floren et al., 2002; Philpott et al., 2004). Neste estudo, Camponotus sp. 1 foi a espécie mais abundante, perfazendo $16,87 \%$ de todos os insetos amostrados, e foi predominante na primavera (Tab. II). O gênero é encontrado com bastante frequiência em uma grande diversidade de hábitats, tendo sido registrado em abundância em eucaliptais (MARINHO et al., 2002), copas de palmeiras em locais alagados (Battirola et al., 2005), ecossistemas degradados (LUTINSKI \& GARCIA, 2005) e florestas em diferentes estágios de ocupação humana (FLOREN et al., 2001).

Os coleópteros destacaram-se pelo maior número de espécies registradas (Tab. I), incluindo a segunda espécie mais abundante, Coccidophilus sp. $(11,85 \%)$ (Coccinellidae). O gênero é bem representado no citros por Coccidophilus citricola Brèthes, 1905, a qual é relatada como predadora de diaspidídeos (Hemiptera, Sternorrhyncha) (PAIVA et al., 1994) e de ocorrência generalizada nos ecossistemas cítricos brasileiros (SILVA et al., 2005). Coccinellidae abrange espécies de hábito diversificado, sendo algumas fitófagas, outras micófagas, porém a maioria é predadora (ZAHOOR et al., 2003). Estão presentes em pomares de citros (STUART et al., 2002), alimentando-se comumente de pulgões, cochonilhas (Michaud, 2001; Silva et al., 2001) e ácaros (VILlanueva et al., 2004). No Rio Grande do Sul, ARIOLI \& LiNK (1987), em levantamento de Coccinellidae no citros, verificaram maior número de espécimes durante os meses de julho e agosto, atribuindo este fato à grande abundância de presas existentes no pomar neste período. No presente trabalho, os coccinelídeos foram mais abundantes no outono e inverno, quando mais de $70 \%$ dos indivíduos desta família foram coletados. A abundância também pode

Tabela I. Ordem, número de famílias e de espécies/morfoespécies, número total e porcentagem de insetos predadores amostrados em pomar orgânico de Citrus deliciosa var. Montenegrina, Montenegro, RS, março de 2004 a março de 2005.

\begin{tabular}{|c|c|c|c|}
\hline Ordens & Famílias & $\begin{array}{c}\text { Espécies/ } \\
\text { morfoespécies }\end{array}$ & Indivíduos $(\%)$ \\
\hline Coleoptera & 3 & 23 & $218(33,1)$ \\
\hline Hymenoptera & 1 & 9 & $337(51,2)$ \\
\hline Neuroptera & 2 & 10 & $74(11,2)$ \\
\hline Thysanoptera & 2 & 4 & $22 \quad(3,3)$ \\
\hline Hemiptera & 2 & 5 & $7 \quad(1,1)$ \\
\hline Total & 10 & 51 & 658 \\
\hline
\end{tabular}


Tabela II. Abundância sazonal e total (N), abundância relativa (ar), constância (C) e riqueza (S) de insetos predadores em pomar orgânico de Citrus deliciosa var. Montenegrina, Montenegro, RS, março de 2004 a março de 2005 (Ac, acessória; Ad, acidental; Ct, constante).

\begin{tabular}{|c|c|c|c|c|c|c|c|}
\hline Espécies/Morfoespécies & Outono & Inverno & Primavera & Verão & $\mathrm{N}$ & ar & $\mathrm{C}$ \\
\hline \multicolumn{8}{|l|}{ COLEOPTERA } \\
\hline \multicolumn{8}{|l|}{ Coccinellidae } \\
\hline Brachiacantha sp. & 4 & 1 & 4 & 0 & 9 & 1,37 & $\mathrm{Ad}$ \\
\hline Chilicorus sp. & 0 & 1 & 0 & 0 & 1 & 0,15 & Ad \\
\hline Calloeneis signata (Korchefsky) & 3 & 1 & 1 & 0 & 5 & 0,76 & $\mathrm{Ac}$ \\
\hline Coccidophilus sp. & 37 & 31 & 5 & 5 & 78 & 11,85 & $\mathrm{Ct}$ \\
\hline Cycloneda pulchella (Klug, 1829) & 0 & 3 & 9 & 1 & 13 & 1,98 & $\mathrm{Ac}$ \\
\hline Cycloneda sanguinea (Linnaeus, 1763) & 0 & 3 & 4 & 0 & 7 & 1,06 & $\mathrm{Ac}$ \\
\hline Delphastus sp. & 7 & 12 & 2 & 0 & 21 & 3,19 & $\mathrm{Ac}$ \\
\hline Exochomus orbiculus Weise, 1893 & 2 & 0 & 1 & 3 & 6 & 0,91 & $\mathrm{Ac}$ \\
\hline Hyperaspis sp. & 6 & 4 & 1 & 0 & 11 & 1,67 & $\mathrm{Ac}$ \\
\hline Stethorus sp. & 6 & 1 & 0 & 8 & 15 & 2,28 & $\mathrm{Ac}$ \\
\hline Scymnus sp. 1 & 1 & 0 & 0 & 0 & 1 & 0,15 & Ad \\
\hline Scymnus sp. 2 & 1 & 0 & 0 & 0 & 1 & 0,15 & $\mathrm{Ad}$ \\
\hline Scymnus sp. 3 & 0 & 1 & 0 & 0 & 1 & 0,15 & $\mathrm{Ad}$ \\
\hline Scymnus sp. 4 & 0 & 1 & 0 & 0 & 1 & 0,15 & $\mathrm{Ad}$ \\
\hline Scymnus sp. 5 & 0 & 1 & 0 & 0 & 1 & 0,15 & Ad \\
\hline Symninae indet. 1 & 2 & 5 & 0 & 0 & 7 & 1,06 & Ad \\
\hline Symninae indet. 2 & 18 & 5 & 2 & 1 & 26 & 3,95 & $\mathrm{Ct}$ \\
\hline Symninae indet. 3 & 0 & 1 & 0 & 0 & 1 & 0,15 & Ad \\
\hline Symninae indet. 4 & 0 & 0 & 0 & 1 & 1 & 0,15 & $\mathrm{Ad}$ \\
\hline Ortalini indet. & 1 & 1 & 0 & 0 & 2 & 0,30 & Ad \\
\hline \multicolumn{8}{|l|}{ Staphylinidae } \\
\hline Palamimus sp. & 0 & 1 & 1 & 0 & 2 & 0,30 & $\mathrm{Ad}$ \\
\hline Stenus bruchi & 2 & 1 & 4 & 0 & 7 & 1,06 & $\mathrm{Ac}$ \\
\hline \multicolumn{8}{|l|}{ Carabidae } \\
\hline Lebiini & 1 & 0 & 0 & 0 & 1 & 0,15 & $\mathrm{Ad}$ \\
\hline \multicolumn{8}{|l|}{ HYMENOPTERA } \\
\hline \multicolumn{8}{|l|}{ Formicidae } \\
\hline Brachymyrmex heeri Forel, 1874 & 2 & 1 & 42 & 11 & 56 & 8,51 & $\mathrm{Ct}$ \\
\hline Camponotus grupo crassus & 22 & 11 & 11 & 23 & 67 & 10,18 & $\mathrm{Ct}$ \\
\hline Camponotus sp. 1 & 31 & 19 & 39 & 22 & 111 & 16,87 & $\mathrm{Ct}$ \\
\hline Camponotus sp. 2 & 0 & 0 & 1 & 0 & 1 & 0,15 & $\mathrm{Ad}$ \\
\hline Crematogaster sp. 1 & 5 & 8 & 2 & 30 & 45 & 6,84 & $\mathrm{Ct}$ \\
\hline Crematogaster sp. 2 & 9 & 3 & 13 & 2 & 27 & 4,10 & $\mathrm{Ct}$ \\
\hline Pheidole falax & 3 & 3 & 7 & 3 & 16 & 2,43 & $\mathrm{Ct}$ \\
\hline Pheidole sp.1 & 1 & 0 & 3 & 7 & 11 & 1,67 & $\mathrm{Ac}$ \\
\hline Wasmannia sp. & 0 & 0 & 1 & 2 & 3 & 0,46 & Ad \\
\hline \multicolumn{8}{|l|}{ NEUROPTERA } \\
\hline \multicolumn{8}{|l|}{ Chrysopidae } \\
\hline Ceraeochrysa sp. 1 & 0 & 0 & 4 & 4 & 8 & 1,22 & $\mathrm{Ac}$ \\
\hline Ceraeochrysa sp. 2 & 0 & 0 & 1 & 0 & 1 & 0,15 & Ad \\
\hline Ceraeochrysa sp. 3 & 0 & 0 & 4 & 4 & 8 & 1,22 & Ad \\
\hline Ceraeochrysa cincta (Schneider,1851) & 1 & 2 & 3 & 16 & 22 & 3,34 & $\mathrm{Ct}$ \\
\hline Ceraeochrysa claveri (Navas, 1911) & 7 & 2 & 1 & 5 & 15 & 2,28 & $\mathrm{Ct}$ \\
\hline Ceraeochrysa cubana (Hagen, 1861) & 0 & 0 & 0 & 1 & 1 & 0,15 & Ad \\
\hline Ceraeochrysa smithi (Navas, 1914) & 0 & 0 & 1 & 0 & 1 & 0,15 & Ad \\
\hline Chrysoperla externa (Hagen, 1861) & 0 & 0 & 3 & 0 & 3 & 0,46 & $\mathrm{Ad}$ \\
\hline Chrysopidae indet. & 0 & 3 & 1 & 1 & 5 & 0,76 & $\mathrm{Ac}$ \\
\hline \multicolumn{8}{|l|}{ Hemerobiidae } \\
\hline Hemerobiidae indet. & 0 & 0 & 10 & 0 & 10 & 1,52 & Ad \\
\hline \multicolumn{8}{|l|}{ THYSANOPTERA } \\
\hline \multicolumn{8}{|l|}{ Aeolothripidae } \\
\hline Franklinothrips tenuicornis Hood, 1915 & 1 & 1 & 1 & 1 & 4 & 0,61 & $\mathrm{Ac}$ \\
\hline Phlaeothripidae & & & & & & & \\
\hline Karnyothrips venustus (Moulton, 1941) & 1 & 5 & 3 & 0 & 9 & 1,37 & $\mathrm{Ac}$ \\
\hline Karnyothrips sp. 1 & 0 & 0 & 2 & 4 & 6 & 0,91 & $\mathrm{Ac}$ \\
\hline Karnyothrips sp. 2 & 0 & 1 & 2 & 0 & 3 & 0,46 & Ad \\
\hline HEMIPTERA & & & & & & & \\
\hline Miridae & & & & & & & \\
\hline Phytocoris sp. & 1 & 0 & 0 & 0 & 1 & 0,15 & Ad \\
\hline Reduviidae & & & & & & & \\
\hline Heza ventralis Stål, 1872 & 1 & 0 & 0 & 0 & 1 & 0,15 & Ad \\
\hline Empicoris rubromaculatus Blackburn, 1889 & 0 & 0 & 3 & 0 & 3 & 0,46 & Ad \\
\hline Zelus sp. & 0 & 0 & 1 & 0 & 1 & 0,15 & Ad \\
\hline Harpactorinae indet. & 0 & 1 & 0 & 0 & 1 & 0,15 & $\mathrm{Ad}$ \\
\hline $\mathrm{N}$ & 176 & 134 & 193 & 155 & 658 & & \\
\hline $\mathrm{S}$ & 27 & 31 & 35 & 22 & 51 & & \\
\hline
\end{tabular}


ter sido decorrência da disponibilidade de alimento, já que inúmeras espécies de fitófagos associados ao citros são referidas como presas de coccinelídeos. WolfF et al. (2004), ao estudarem a ação de predação de coccinelídeos sobre diaspidídeos (Sternorrhyncha) em pomar de Citrus sinensis (Linnaeus) Osbeck, na mesma região do presente estudo, verificaram maior ocorrência das presas nos meses de março a agosto. Como pulgões são mais comumente relacionados às brotações de citros e aos períodos mais quentes do ano, acredita-se que estes coccinelídeos tenham sido mantidos pela disponibilidade de cochonilhas. Scymnus Kugelann, 1794 (Coccinellidae) foi o gênero que apresentou maior número de morfoespécies. Espécies de Scymnus também têm sido registradas em pomares cítricos de outras regiões do Rio Grande do Sul (Arioli \& Link, 1987) e do Estado de São Paulo (Lioni \& Cividanes, 2004).

Entre os neurópteros, foram considerados apenas os indivíduos no estágio imaturo, já que, além dos adultos serem de difícil captura com este método, quase a totalidade das espécies deste grupo apresenta o hábito predador na fase larval (TRIPLEHORN \& JOHNSON, 2005). Chrysopidae, em especial, abriga espécies reconhecidas pelo importante papel no controle de artrópodes, além de possuir uma elevada adaptabilidade a diferentes ambientes. Esta característica lhes permite ter uma ampla distribuição geográfica (GITIRANA-NETO et al., 2001), com ocorrência em ecossistemas naturais e implantados. No citros, contam com um grande número de presas, como cochonilhas, mosca-branca, ácaros e larvas do minador-docitros, Phyllocnistis citrella Stainton, 1856 (Lepidoptera, Gracillariidae) (FreITAS, 2002), importantes pragas na cultura (URBANEJA et al., 2001). Entre os crisopídeos encontrados, Ceraeochrysa Adams, 1982 foi o gênero mais abundante. Este neuróptero também foi registrado em pomares cítricos da região de Lavras, Minas Gerais (Vezon \& CARVALHO, 1993), onde apresentou o pico de ocorrência nos meses de baixa temperatura e precipitação (GITIRANA-NETO et al., 2001), diferentemente do encontrado no presente estudo quanto à temperatura, pois a maior incidência de indivíduos do gênero foi verificada durante o verão. No entanto, nesta estação também foram verificados os menores índices de precipitação pluviométrica do período de amostragem, podendo ser este um dos fatores que influenciaram na abundância do grupo.

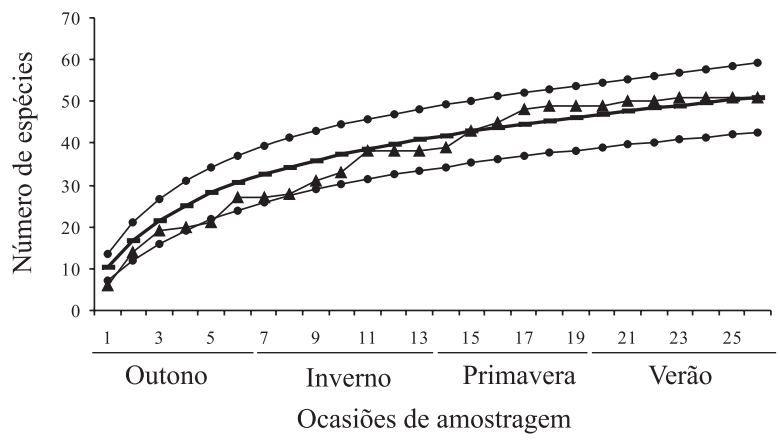

Fig. 1. Curva de suficiência amostral de insetos, em dossel de Citrus

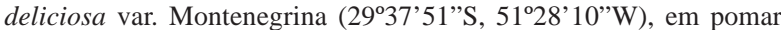
orgânico, Montenegro, RS, março de 2004 a março de 2005 (-•intervalo de confiança; - $\mathbf{\Delta}$ - número acumulado de espécies observado; -- número acumulado de espécies corrigido).
Em relação a Thysanoptera, apesar de Franklinothrips Back, 1912 não ter sido muito abundante, este constitui um dos poucos gêneros cujas espécies são, provavelmente, todas predadoras de pequenos artrópodes, tanto na fase larval como na adulta, sendo algumas destas usadas como agentes de controle biológico (Mound \& Reynaud, 2005). No Brasil, $F$. tenuicornis Hood, 1915 já foi referida por SILVA et al. (1968) em folhas de cafeeiro em Minas Gerais. Este foi o primeiro registro da espécie no Rio Grande do Sul.

Hemiptera foi o grupo que apresentou menor abundância de indivíduos (sete), representados quase que exclusivamente por reduviídeos. Dentre as espécies de Reduviidae amostradas, as dos gêneros Zelus Fabricius, 1803 e Heza Amyot \& Serville, 1843 já haviam sido referidas por PARRA et al. (2003) em pomares cítricos de São Paulo. LiONI \& CIVIDANES (2004), em trabalho desenvolvido em pomares de citros naquele mesmo estado, apontaram indivíduos de Zelus sp. como responsáveis pela predação em ovos do minador-dos-citros, exercendo um importante papel como inimigo natural. Na Costa Rica, Elzzondo Solis (2002) registrou que a população de Zelus sp. foi a mais abundante dentre os hemípteros.

Analisando-se sazonalidade, a maior abundância de indivíduos $(29,33 \%)$ foi constatada durante a primavera, sem, no entanto, haver diferença significativa $(p=0,30)$ entre as estações. O maior número de predadores na primavera pode estar relacionado a dois fatores. Nesta estação ocorre o principal fluxo de brotação no citros (Koller, 1994), disponibilizando grande quantidade de recurso alimentar atrativo para diversas espécies fitófagas, como $P$. citrella, pulgões (T. citricida) e cochonilhas (Coccus viridis Green,1889), grupos comumente encontrados nos brotos da planta (PARRA et $a l ., 2003)$ e que são presas potenciais para a manutenção da comunidade de predadores. Adicionalmente, fatores abióticos como a temperatura - que a partir da primavera sofre elevação - propiciam condições favoráveis ao crescimento, reprodução e a dispersão dos insetos de um modo geral (SPEIGHT et al., 1999).

$\mathrm{Na}$ avaliação da diversidade da entomofauna amostrada, analisando-se as curvas de rarefação (Fig. 2), verifica-se que, para uma subamostra de 131 indivíduos, não houve diferença significativa quanto à riqueza

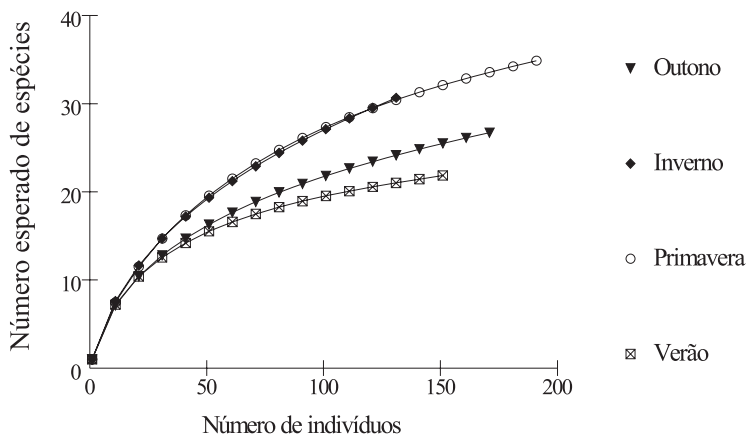

Fig. 2. Curvas de rarefação de insetos coletados em pomar orgânico de Citrus deliciosa var. Montenegrina (29³7'51'S, 51²8'10"W), Montenegro, RS, de março de 2004 a março de 2005. 

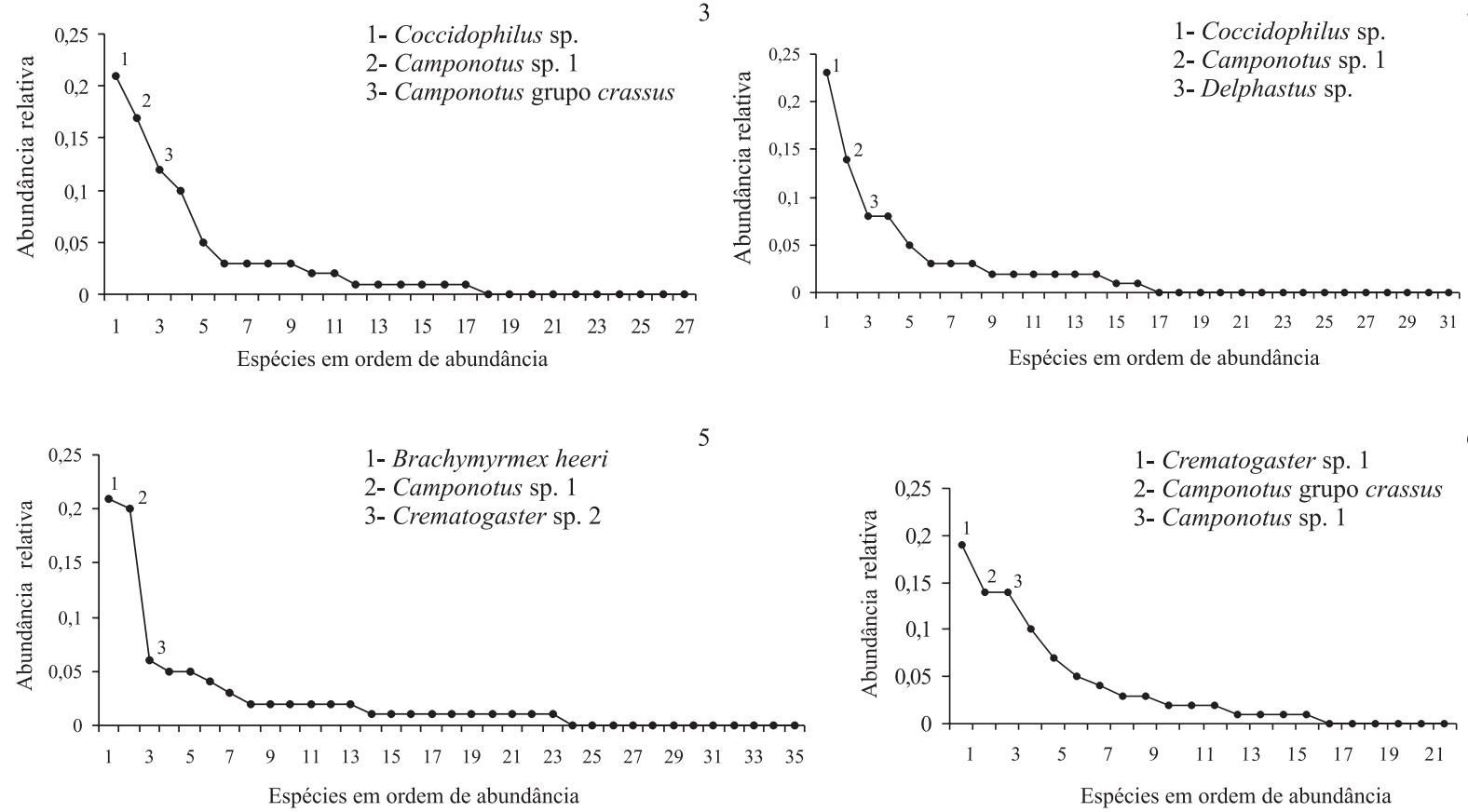

4

6

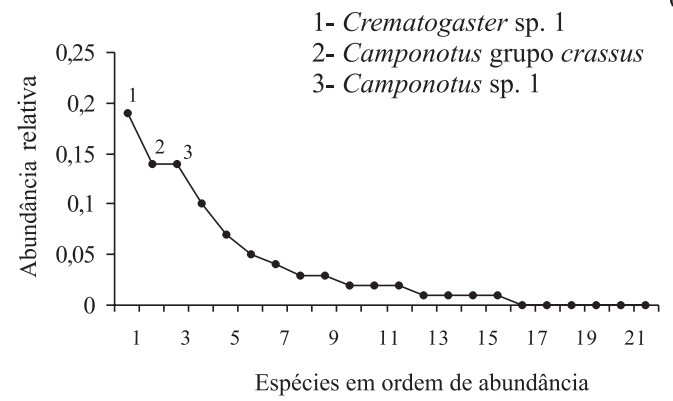

Figs. 3-6. Distribuição da abundância relativa das espécies de insetos amostrados em pomar orgânico de Citrus deliciosa var. Montenegrina

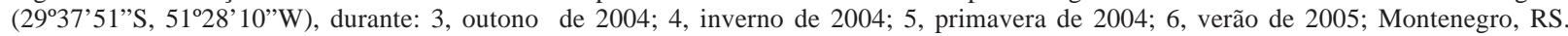

estimada de espécies $\left(\mathrm{S}_{\mathrm{est}} \pm \mathrm{dp}\right)$, entre o inverno $(30,66 \pm$ $0,54)$ e a primavera $(30,42 \pm 1,72)$, embora nesta última tenha-se registrado o maior número de indivíduos. $\mathrm{O}$ outono $(24,15 \pm 1,42)$ e o verão $(21,02 \pm 0,90)$ diferiram entre si quanto à riqueza estimada, e foram significativamente menos ricos do que o inverno e primavera. Em relação à distribuição da abundância (Figs. 3-6), os valores máximos de abundância foram semelhantes em todas as estações (próximos de 20\%). Quanto à constância observada, dez espécies apresentaram-se constantes, 14 acessórias e as demais acidentais (Tab. II). Camponotus sp. 1 (Hymenoptera: Formicidae), Coccidophilus sp. (Coleoptera: Coccinellidae) e Camponotus grupo crassus foram dominantes em relação às demais espécies.

Não houve correlação significativa entre a flutuação da entomofauna com precipitação $(p=0,89)$ e temperatura média $(\mathrm{p}=0,74)$; entretanto, a baixa abundância e riqueza de predadores registradas no verão podem ter sido efeito indireto da baixa precipitação pluviométrica ocorrida de dezembro a fevereiro. Conforme Berlato (2005), esta foi uma das maiores estiagens verificada nos últimos 50 anos no Rio Grande do Sul, quando choveu apenas $20 \%$ do esperado para os meses de dezembro a fevereiro. Assim, a estiagem pode ter influenciado a abundância de predadores por afetar a qualidade do recurso para as comunidades de insetos fitófagos e, conseqüentemente, a disponibilidade de presas.

A vegetação cultivada geralmente apresenta riqueza e abundância de fauna reduzidas, se comparada àquelas encontradas em um ambiente natural (GILLER et al., 1997), em grande parte por ser constituída por uma arquitetura vegetal única. Este estudo, entretanto, evidencia a grande diversidade de artrópodes predadores no agroecossistema citros, fornecendo subsídios para o manejo e conservação destas espécies.

Agradecimentos. Aos pesquisadores M.Sc. Luciano de Azevedo Moura (FZBRS, Porto Alegre), Dra. Lúcia Almeida (UFPR, Curitiba), Dra. Natalia J. Vandenberg, Systematic Entomology Laboratory, Agricultural Research Service, US Department of Agriculture (USDA, Washington, D.C., EUA), Dr. Volker Puthz (Max-Planck-Institut für Limnologie, Plön, Alemanha), Dr. Michel Brancucci (Natural History Museum, Basel, Suíça), Dr. Diego Carpintero (Faculdad de Ciencias Naturales y Museo de La Plata, La Plata, Argentina), Dr. Paulo Sergio Fiuza Ferreira (UFV, Viçosa), Dr. Hélcio Gil-Santana (FIOCRUZ, Rio de Janeiro), Dra. Elena Diehl (UNISINOS, São Leopoldo), Dr. Antonio Mayhé Nunes (UFRRJ, Seropédica), M.Sc. Adriano Cavalleri e Dra. Silvia Pinent (UFRGS, Porto Alegre) e Dr. Gilberto Albuquerque (UENF, Campos dos Goytacazes), pela colaboração na identificação do material coletado. Ao $\mathrm{CNPq}$, pela concessão de bolsas de Mestrado e de Produtividade ao primeiro e terceiro autores, respectivamente.

\section{REFERÊNCIAS BIBLIOGRÁFICAS}

ARIoli, M. C. S. \& LinK, D. 1987. Ocorrência de joaninhas em pomares cítricos na região de Santa Maria, RS. Revista Centro de Ciências Rurais 17(3):213-222.

Battirola, L. D.; Maroues, M. I.; Adis, J. \& Delabie, J. H. C. 2005. Composição da comunidade de Formicidade (Insecta, Hymenoptera) em copas de Attalea phalerata Mart. (Arecaceae), no Pantanal de Poconé, Mato Grosso, Brasil. Revista Brasileira de Entomologia 49(1):107-117.

Berlato, M.A. 2005. Prognósticos e recomendações para o período de julho/agosto/setembro 2005. Disponível em: <http://www.emater.tche.br/site/destaques/ptbr/pdf/ forum tempoclima/reuniaocopaaergs 7-5-07-2005.pdf>. Acesso em: 7.12.2005.

Berryman, A. A. \& Guttierrez, A. P. 1999. Dynamics of insect predator-prey interation. In: Huffaker, C. B. \& Guttierrez, A P. eds. Ecological Entomology. New York, John Wiley \& Sons. p.389-424. 
Berti Filho, E. \& Ciociola, A. I. 2002. Parasitóides ou predadores? Vantagens e desvantagens. In: PARRA, J. R. P.; Botelho, P. S. M.; Corrêa-Ferreira, B. S. \& Bento, J. M. S. eds. Controle biológico no Brasil: parasitóides e predadores. São Paulo, Manole. p.29-41.

Colwell, R. K. 2005. EstimateS: Statistic estimation of species richness and shared species from samples. Version 7.5. Disponível em: 〈http://viceroy.eeb.uconn.edu/estimates>. Acesso em: 04.01.2006.

Elizondo Solis, J. M. 2002. Inventario y flutuación poblacional de insectos y arañas asociadas con Citrus sinensis en la región Huetar norte da Costa Rica. Manejo Integrado de Plagas y Agroecologia 64:88-98.

Floren, A.; Freking, A.; Biehl, M. \& Linsenmair, K. E. 2001. Anthropogenic disturbance changes the structure of arboreal tropical ant communities. Ecography 24:547-554.

2002. Arboreal ants as key predators in tropical lowland rainforest trees. Oecologia 131:137-144.

Freitas, S. 2002. O uso de crisopídeos no controle biológico de pragas. In: Parra, J. R. P.; Botelho, P. S. M.; CorrêA-Ferreira, B. \& Bento, J. M. S. eds. Controle biológico no Brasil, parasitóides e predadores. São Paulo, Manole. p.209-219.

Giller, K.; Beare, M. H.; Lavelle, P.; Izac, A. M. N. \& Swift, M. 1997. Agricultural intensification, soil biodiversity and agroecosystem function. Applied Soil Ecology 6:3-16.

Gitirana Neto, J.; Carvalho, C. F.; Souza, B. \& Santa-Cecília, L. V. C. 2001. Flutuação de espécies de Ceraeochrysa Adams, 1982 (Neuroptera: Chrysopidae) em citros, na região de Lavras MG. Ciência e Agrotecnologia 25(3):550-559.

Hammer, O.; Harper, D. A. T. \& Rian, P. D. 2001. Past: Palaeonthological statistics software package for education and data analysis. Version. 1.37. Disponível em: <http:// palaeo-electronica.org/2001_1/past/issue1_01.htm>. Acesso em: 12.11.2005.

Holldobler, B. \& Wilson, E. O. 1990. The ants. Cambridge, Harvard University. 772p.

IPAGRO (Instituto de Pesquisas Agronômicas). 1989. Atlas Agroclimático do Estado do Rio Grande do Sul. Porto Alegre, IPAGRO. 102p

Koller, O. C. 1994. Citricultura: laranja, limão e tangerina Porto Alegre, Rigel. 446p.

Lara, F. M.; Bortoli, S. A. \& Oliveira, E. A. 1977. Flutuações populacionais de alguns insetos associados ao Citrus sp. e suas correlações com atores meteorológicos. Científica 5(2): 134-143.

Lioni, A. S. R. \& Cividanes, F. J. 2004. Tabela ecológica do minador-dos-citros, Phyllocnistis citrella Stainton (Lepidoptera: Gracillariidae). Neotropical Entomology 33(4):407-415.

Lutinski, J. A. \& Garcia, F. R. M. 2005. Análise faunística de Formicidae (Hymenoptera: Apocrita) em ecossistema degradado no município de Chapecó, Santa Catarina. Biotemas 18(2):73-86.

MagurRan, A. E. 2004. Measuring biological diversity. Oxford, Blackwell. 256p.

Marinho, C. G. S.; Zanetti, R.; Delabie, J. H. C.; Schelindwein, M. N. \& Ramos, L. S. 2002. Diversidade de formigas (Hymenoptera: Formicidae) da serrapilheira em eucaliptais (Myrtaceae) e área de serrado de Minas Gerais. Neotropical Entomology 31(2): 187-195.

McAleece, N. 1997. Biodiversity Professional 2.0. The Natural History Museum and the Scotish Association for Marine Science. Disponível em: <http://www.nhm.ac.uk/ zoology/bdpro>. Acesso em: 12.12.2005.

Michaud, J. P. 2001. Numerical response of Olla v-nigrum (Coleoptera: Coccinellidae) to infestations of Asian in citrus psyllid (Hemiptera: Psyllidae) in Florida. Florida Entomologist 84(4):608-612.
Moreno, C. E. 2001. Métodos para medir la biodiversidad. Zaragoza, Cited/ Unesco \& SEA. v. 1, 84p. (Série Manuales y Tesis SEA).

Mound, L. \& Reynaud, P. 2005. Franklinothrips, a pantropical Thysanoptera genus of ant-mimicking obligate predators (Aeolothripidae). Zootaxa 864:1-16.

Paiva, P. E. B.; Silva, J. L.; Yamamoto, P. T. \& Gravena, S. 1994 A entomofauna da planta cítrica na região de Jaboticabal (SP). Laranja 15(1):295-311.

Parra, J. R. P.; Negri, H. O. \& Pinto, A. S. 2003. Guia ilustrado de pragas e insetos benéficos dos citros. Piracicaba, A. S Pinto. 140p.

Pedigo, L. P. 1996. Entomology and pest management. 2 ed Upper Saddle River, Prentice Hall. 679p.

Philpott, S. M.; Greenberg, R. \& Bichier, P. 2004. Impacts of major predators on tropical agroforest arthropods: comparisons within and across taxa. Oecologia 140: 140-149.

Ricklefs, R. E. \& Miller, G. L. 1999. Ecology. New York, W. H. Freeman and Company. 822p.

Silva, R. A.; Almeida, L. M. de \& Busoli, A. C. 2005. Morfologia dos imaturos e do adulto de Coccidophilus citricola Brèthes (Coleoptera, Coccinellidae, Sticholotininae), predador de cochonilhas-de-carapaça (Hemiptera, Diaspididae) de citros. Revista Brasileira de Entomologia 49(1):29-35.

Silva, D. C.; Cordeiro, E. O. \& Corseuil, E. 2001. Levantamento de coccinelídeos (Coleoptera, Coccinellidae) predadores em plantas cítricas. Pesquisa Agropecuária Gaúcha 7(1): $105-110$.

Silva, A. G. A.; Gonçalvez, C. R.; Galvão, D. M.; Gonçalvez, A. J. L.; Gomes, J.; Silva, M. N. \& Simoni, L. 1968. Quarto catálogo dos insetos que vivem nas plantas do Brasil seus parasitos e predadores. Rio de Janeiro, Ministério da Agricultura. 622p.

Silveira-Neto, S.; Nakano, O.; Barbin, D. \& Villa Nova, N. A. 1976. Manual de ecologia dos insetos. São Paulo, Agronômica Ceres. 419p

Speight, M. R.; Hunter, M. D. \& Watt, A. D. 1999. Ecology of insects: concepts and applications. London, Blackwell Science. 350p.

Stuart, R. J.; Michaud, J. P.; Olsen, L. \& McCoy, C. W. 2002 Ladybeetles as potential predators of the root weevil Diaprepes abbreviatus (Coleoptera: Curculionidae) in Florida citrus. Florida Entomologist 85(3):9-15

Triplehorn, C. A. \& Johnson, N. F. 2005. Borror \& DeLong's introduction to the study of insects. $7 \mathrm{ed}$. Belmont, Brooks/Cole. 864p.

Urbaneja, A.; MuÑoz, A.; Garrido, A. \& Jacas, A. 2001. Incidencia de Chrysoperla carnea (Stephens) (Neuroptera: Chrysopidae) em la depredation de Phyllocnistis citrella. Boletin de Sanidad Vegetal, Plagas 27:65-73.

Vezon, M. \& Carvalho, C. F. 1993. Desenvolvimento larval, prépupal e pupal de Ceraeochrysa cubana (Hagen) (Neuroptera: Chrysopidae) em diferentes dietas e temperaturas. Anais da Sociedade Entomológica do Brasil 22(2):477-483.

Villanueva, R. T.; Michaud, J. P. \& Childers, C. C. 2004. Ladybeetles as predators of pest and predacious mites en citrus. Journal of Entomologist Science 39(1):23-29.

Wolff, V. R. S.; Pulz, C. E.; Silva, D. C.; Mezzomo, J. B. \& Prade, C. A. 2004. Inimigos naturais associados a Diaspididae (Hemiptera, Sternorrhyncha), ocorrentes em Citrus sinensis (Linnaeus) Osbeck, no Rio Grande do Sul, Brasil: I - Joaninhas e fungos entomopatógenos. Arquivos do Instituto Biológico 71(3):355-361.

Zahoor, M. K.; Suhail, A.; Igbal, J.; Zulfagar, Z. \& Anwar, M. 2003. Biodiversity of predaceous coccinellids and their role as bioindicators in an agro-ecosystem. International Journal for Agriculture and Biology 5(4):555-559. 